九州大学学術情報リポジトリ

Kyushu University Institutional Repository

\title{
A computer-assisted instability proof for the Orr-Sommerfeld problem with Poiseuille flow
}

Watanabe, Yoshitaka

Research Institute for Information Technology, Kyushu University

Plum, Michael

Faculty of Mathematics, Karlsruhe University

Nakao, Mitsuhiro T.

Faculty of Mathematics, Kyushu University

http://hdl. handle. net/2324/8826

出版情報 : 2008-01-21. 九州大学大学院数理学研究院 バージョン：

権利関係 : 


\section{MHF Preprint Series}

Kyushu University 21st Century COE Program

Development of Dynamic Mathematics with High Functionality

\section{A computer-assisted instability proof for the Orr-Sommerfeld problem with Poiseuille flow}

\section{Y. Watanabe, M. Plum \\ M.T. Nakao}

MHF 2008-3

( Received January 21, 2008 )

Faculty of Mathematics

Kyushu University

Fukuoka, JAPAN 


\title{
A computer-assisted instability proof for the Orr-Sommerfeld problem with Poiseuille flow
}

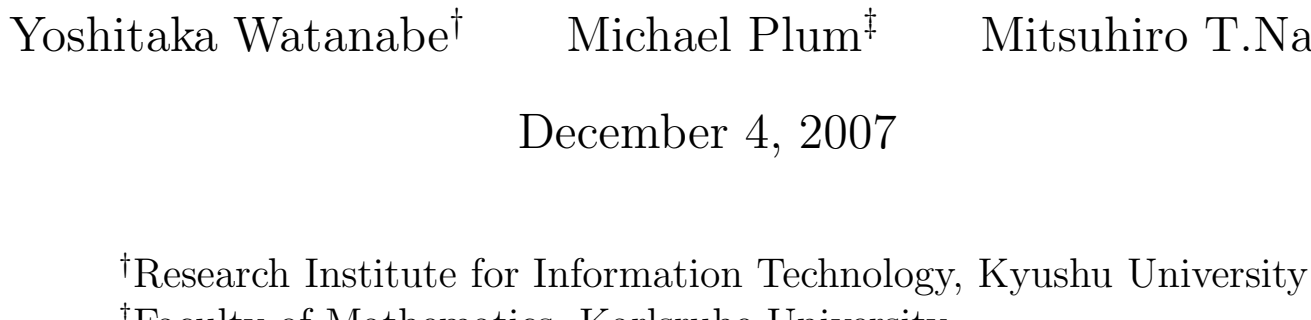 \\ ${ }^{\ddagger}$ Faculty of Mathematics, Karlsruhe University \\ *Faculty of Mathematics, Kyushu University
}

\begin{abstract}
This paper presents a computer-assisted proof of solutions of the Orr-Sommerfeld equation describing hydrodynamic stability of Poiseuille flow. A numerical verification method for computing eigenpair enclosures for this non-selfadjoint eigenvalue problem is described. Some verification results confirm the effectiveness of the method.
\end{abstract}

\section{The Orr-Sommerfeld model}

Consider a two-dimensional flow of an incompressible viscous fluid between two infinite parallel plates at $y=y_{1}$ and $y=y_{2}$ (See Figure 1). The flow between the parallel plates

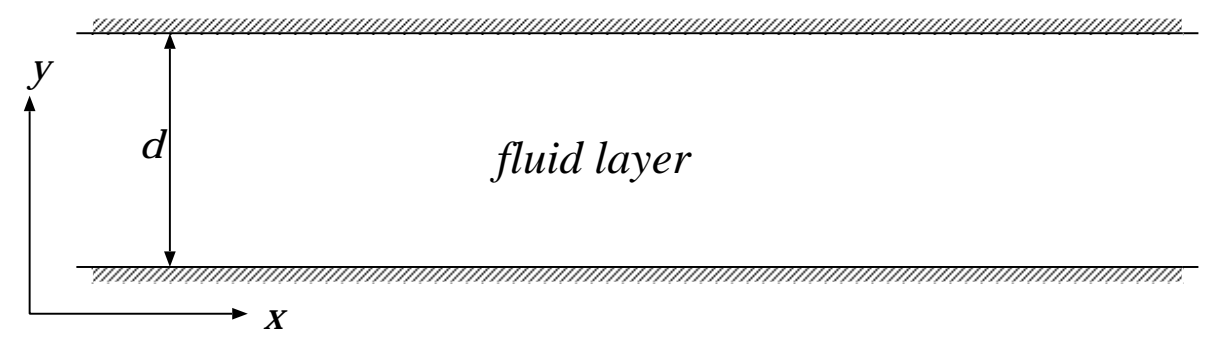

Figure 1: infinite parallel plates; $d:=y_{2}-y_{1}$

is described by the unsteady nonlinear incompressible non-dimensionalized Navier-Stokes equations:

$$
\left\{\begin{aligned}
\frac{\partial u}{\partial t}+u \frac{\partial u}{\partial x}+v \frac{\partial u}{\partial y} & =-\frac{\partial p}{\partial x}+\frac{1}{R} \Delta u \\
\frac{\partial v}{\partial t}+u \frac{\partial v}{\partial x}+v \frac{\partial v}{\partial y} & =-\frac{\partial p}{\partial y}+\frac{1}{R} \Delta v \\
\frac{\partial u}{\partial x}+\frac{\partial v}{\partial y} & =0
\end{aligned}\right.
$$


where, $u, v$ and $p$ are the velocity in the horizontal direction, the velocity in the vertical direction and the pressure field respectively, and $R$ is the Reynolds number.

Let the basic (primary) flow be represented by

$$
(u, v)=(U(y), 0), \quad p=p_{0}+\frac{1}{R} \frac{d^{2} U(y)}{d y^{2}} x, \quad 0 \leq x<\infty, \quad y_{1} \leq y \leq y_{2} .
$$

Here, $p_{0}$ is a constant and $U$ is a quadratic polynomial in $y$.

In order to study the linear stability of the system (1), we consider a small perturbation $(\hat{u}, \hat{v}, \hat{p})$ from the basic flow $(2)$ such that

$$
u=U+\hat{u}, \quad v=\hat{v}, \quad p=P+\hat{p},
$$

where $U=U(y)$ and $P=p_{0}+\frac{1}{R} \frac{d^{2} U(y)}{d y^{2}} x$. Substituting for the equation (1) and disregarding the second-order terms involving products of the perturbations, the linearized equations:

$$
\left\{\begin{aligned}
\frac{\partial \hat{u}}{\partial t}+U \frac{\partial \hat{u}}{\partial x}+\hat{v} \frac{d U}{d y} & =-\frac{\partial \hat{p}}{\partial x}+\frac{1}{R} \Delta \hat{u} \\
\frac{\partial \hat{v}}{\partial t}+U \frac{\partial \hat{v}}{\partial x} & =-\frac{\partial \hat{p}}{\partial y}+\frac{1}{R} \Delta \hat{v} \\
\frac{\partial \hat{u}}{\partial x}+\frac{\partial \hat{v}}{\partial y} & =0
\end{aligned}\right.
$$

are obtained.

Next, in order to satisfy the divergence free condition, the stream function $\psi(t, x, y)$ which satisfies

$$
\hat{u}=\frac{\partial \psi}{\partial y}, \quad \hat{v}=-\frac{\partial \psi}{\partial x}
$$

is introduced; note that the domain $(0, \infty) \times\left(y_{1}, y_{2}\right)$ is simply connected.

Cross-differentiating the equation (3) in order to eliminate the pressure term implies

$$
\frac{\partial}{\partial t} \frac{\partial^{2} \psi}{\partial x^{2}}+\frac{\partial}{\partial t} \frac{\partial^{2} \psi}{\partial y^{2}}+U \frac{\partial^{3} \psi}{\partial x \partial y^{2}}+U \frac{\partial^{3} \psi}{\partial x^{3}}=\frac{d^{2} U}{d y^{2}} \frac{\partial \psi}{\partial x}+\frac{1}{R} \Delta^{2} \psi
$$

Here, when we impose a no-slip boundary condition at $y=y_{1}$ and $y=y_{2}$, the stream function $\psi$ satisfies

$$
\frac{\partial \psi}{\partial x}=\frac{\partial \psi}{\partial y}=0, \quad y=y_{1}, y_{2}
$$

In view of the independence of the basic flow $(U(y), 0)$ on $x, t$, it makes sense to look for the following travelling wave form of the disturbance stream function $\psi(t, x, y)$ :

$$
\psi=\psi(t, x, y)=\phi(y) e^{i a(x-c t)} .
$$

Here, $\phi(y)$ and $a>0$ mean the amplitude and wavenumber, respectively, and $c=c_{r}+i c_{i}$ is the complex wave speed; $c_{r}$ represents the speed at which a wave propagates downstream, and $a c_{i}$ characterizes the rate at which the disturbance grows or decays in time. If $c_{i}<0$, then $\psi$ decays (i.e. the flow is stable), and if $c_{i}>0$, then $\psi$ grows (the flow is unstable). 
Substituting eq.(7) into eq.(5), and using $D:=d / d y$, the equation

$$
\frac{1}{R}\left(D^{2}-a^{2}\right)^{2} \phi(y)=i a\left[(U-c)\left(D^{2}-a^{2}\right) \phi(y)-\frac{d^{2} U}{d y^{2}} \phi(y)\right]
$$

is obtained. From the no-slip boundary condition $(6), \phi(y)$ must satisfy

$$
\phi=D \phi=0, \quad y=y_{1}, y_{2} .
$$

This equation (8) is the well-known Orr-Sommerfeld problem derived by Orr [6] and Sommerfeld [9] for the disturbance eigenfunction $\phi(y)$, which in turn depends on the prescribed values of the wave number $a$ and of the Reynolds number $R$.

Rewriting $y \rightarrow x$ and $u:=\phi, \lambda:=i a R c$ we have

$$
\left\{\begin{array}{c}
\left(-D^{2}+a^{2}\right)^{2} u+i a R\left[U\left(-D^{2}+a^{2}\right)+U^{\prime \prime}\right] u=\lambda\left(-D^{2}+a^{2}\right) u \quad \text { on } \Omega=\left[x_{1}, x_{2}\right] \\
u\left(x_{1}\right)=u\left(x_{2}\right)=u^{\prime}\left(x_{1}\right)=u^{\prime}\left(x_{2}\right)=0 .
\end{array}\right.
$$

In this paper, we focus on the case of plane Poiseuille flow [4]

$$
U=V:=1-x^{2}, \quad x_{1}=-1, \quad x_{2}=1 .
$$

The Orr-Sommerfeld equation (9) is a non-selfadjoint eigenvalue problem for the eigenpair $(\lambda, u)$, and within the frame of linearized stability theory, the flow is stable if the spectrum is located in the right complex half-plane, otherwise unstable.

There are many numerical results for the Orr-Sommerfeld equation with Poiseuille flow. For example, Orszag [7] solved it numerically using expansions in Chebyshev polynomials and the $Q R$ matrix eigenvalue algorithm. He computed that the smallest value of $R$ for which an unstable eigenmode exists (critical Reynolds number), according to "numerical evidence", is 5772.22 with $a \in[1.0255,1.0257]$. Klein [1] proposed a method for eigenvalue inclusion using a generalization of Gerschgorin's theorem, however, he imposed some additional assumptions, and numerical results did not take into account effects of rounding error in floating point computation. Lahmann and Plum [2] gave a computer-assisted method for computing rigorous eigenvalue enclosures and applied it to the Orr-Sommerfeld problem with Blasius profile. However, concerning plane Poiseuille flow, a rigorous instability proof has never been given from the mathematical point of view.

In this paper, we propose a numerical verification procedure which encloses an eigenpair of the Orr-Sommerfeld equation with plane Poiseuille flow. The method uses numerical means, but all numerical errors are take into account, and hence the method implies a rigorous proof of all statements made. The method is based on a fixed-point theorem with some Newton-like operator. Especially, we are interested in whether the real part of the enclosed eigenvalue $\lambda$ is negative or not, from the point of view in linearized stability theory.

The paper is organized as follows. In Section 2 we formulate a fixed-point equation in an infinite dimensional function space. Section 3 contains a study of a finite dimensional subspace and some constructive a priori error estimates for a projection onto it. Section 4 is concerned with a practical verification algorithm. In Section 5 we report on some verification results which prove the existence of eigenpairs in the computed regions, and in particular give rigorous instability proofs. 


\section{Fixed-point formulation}

Setting

$$
\tilde{\Delta}:=-D^{2}+a^{2}
$$

and using real valued functions $v, w$ and real values $\sigma, \mu$ such that

$$
\left\{\begin{array}{l}
u=v+i w \\
\lambda=\sigma+i \mu
\end{array}\right.
$$

equation (9) becomes

$$
\left\{\begin{array}{c}
\tilde{\Delta}^{2} v-a R\left(V \tilde{\Delta}+V^{\prime \prime}\right) w=\sigma \tilde{\Delta} v-\mu \tilde{\Delta} w \text { on } \Omega \\
\tilde{\Delta}^{2} w+a R\left(V \tilde{\Delta}+V^{\prime \prime}\right) v=\sigma \tilde{\Delta} w+\mu \tilde{\Delta} v \text { on } \Omega \\
v(-1)=v(1)=v^{\prime}(-1)=v^{\prime}(1)=0 \\
w(-1)=w(1)=w^{\prime}(-1)=w^{\prime}(1)=0
\end{array}\right.
$$

Let $L^{2}(\Omega)$ be the real $L^{2}$ space on $\Omega=(-1,1)$ with the inner product $(\cdot, \cdot)_{L^{2}}$ and the norm $\|v\|:=\sqrt{(v, v)_{L^{2}}},\|v\|_{\infty}:=\operatorname{ess} \sup _{x \in \Omega}|v(x)|$ the $L^{\infty}$-norm on $\Omega$, and for integers $k$, let $H^{k}(\Omega)$ denote the $L^{2}$-Sobolev space of order $k$ on $\Omega$ with the norm $\|v\|_{H^{k}}:=$ $\sqrt{\sum_{j=0}^{k}\left\|d^{j} v / d x^{j}\right\|^{2}}$. Denoting

$$
H_{0}^{2}(\Omega):=\left\{v \in H^{2}(\Omega) \mid v(-1)=v^{\prime}(-1)=v(1)=v^{\prime}(1)=0\right\},
$$

$\|v\|_{\tilde{\Delta}}:=\|\tilde{\Delta} v\|$ is an equivalent norm for $\|v\|_{H^{2}}$ and $(\tilde{\Delta} v, \tilde{\Delta} w)_{L^{2}}$ can be chosen as the inner-product of $H_{0}^{2}(\Omega)$. We define a Banach space $X:=H_{0}^{2}(\Omega) \times H_{0}^{2}(\Omega) \times \mathbb{R} \times \mathbb{R}$ with the norm

$$
\left\|[v, w, \sigma, \mu]^{T}\right\|_{X}:=\sqrt{\|v\|_{\tilde{\Delta}}^{2}+\|w\|_{\tilde{\Delta}}^{2}+\sigma^{2}+\mu^{2}} .
$$

Since $\tilde{\Delta}$ has the properties

$$
\begin{aligned}
& (\tilde{\Delta} v, w)_{L^{2}}=(v, \tilde{\Delta} w)_{L^{2}}, \quad \forall v \in H_{0}^{2}(\Omega), \quad \forall w \in H^{2}(\Omega), \\
& (\tilde{\Delta} v, \tilde{\Delta} w)_{L^{2}}=\left(\tilde{\Delta}^{2} v, w\right)_{L^{2}}, \quad \forall v \in C_{0}^{\infty}(\Omega), \quad \forall w \in H_{0}^{2}(\Omega),
\end{aligned}
$$

we can look for solutions for eq.(12), submitted to additional normalizing conditions for the eigenfunction, in the following weak formulation for $[v, w, \sigma, \mu]^{T} \in X$ :

$$
\left\{\begin{aligned}
(\tilde{\Delta} v, \tilde{\Delta} \xi)_{L^{2}} & =\left(a R\left(V \tilde{\Delta}+V^{\prime \prime}\right) w+\sigma \tilde{\Delta} v-\mu \tilde{\Delta} w, \xi\right)_{L^{2}}, \quad \forall \xi \in H_{0}^{2}(\Omega) \\
(\tilde{\Delta} w, \tilde{\Delta} \eta)_{L^{2}} & =\left(-a R\left(V \tilde{\Delta}+V^{\prime \prime}\right) v+\sigma \tilde{\Delta} w+\mu \tilde{\Delta} v, \eta\right)_{L^{2}}, \quad \forall \eta \in H_{0}^{2}(\Omega) \\
\left(v, v_{0}\right)_{L^{2}} & =\xi_{R} \\
\left(w, w_{0}\right)_{L^{2}} & =\xi_{I}
\end{aligned}\right.
$$

where $a, R, \xi_{R}, \xi_{I} \in \mathbb{R}$ and $v_{0}, w_{0} \in H_{0}^{2}(\Omega)$ are given. 
Let bounded continuous maps $f_{1}, f_{2}$ from $X$ to $L^{2}(\Omega)$ be denoted by

$$
\begin{aligned}
& f_{1}[v, w, \sigma, \mu]^{T}:=a R\left(V \tilde{\Delta}+V^{\prime \prime}\right) w+\sigma \tilde{\Delta} v-\mu \tilde{\Delta} w \\
& f_{2}[v, w, \sigma, \mu]^{T}:=-a R\left(V \tilde{\Delta}+V^{\prime \prime}\right) v+\sigma \tilde{\Delta} w+\mu \tilde{\Delta} v .
\end{aligned}
$$

Also by the Lax \& Milgram Lemma, for any $g \in L^{2}(\Omega)$ there exists a unique solution $\omega \in H^{4}(\Omega) \cap H_{0}^{2}(\Omega)$ satisfying

$$
\tilde{\Delta}^{2} \omega=g
$$

For $g \in L^{2}(\Omega)$ let $\left(\tilde{\Delta}^{2}\right)^{-1} g$ be the solution of eq.(16), then the operator $\left(\tilde{\Delta}^{2}\right)^{-1}$ : $L^{2}(\Omega) \longrightarrow H_{0}^{2}(\Omega)$ is compact due to the compactness of the imbedding $H^{4}(\Omega) \hookrightarrow H_{0}^{2}(\Omega)$.

Using $f_{1}, f_{2}$ and $\left(\tilde{\Delta}^{2}\right)^{-1}$, the operator $F: X \longrightarrow X$ defined by

$$
F[v, w, \sigma, \mu]^{T}:=\left[\begin{array}{c}
\left(\tilde{\Delta}^{2}\right)^{-1} f_{1}[v, w, \sigma, \mu]^{T} \\
\left(\tilde{\Delta}^{2}\right)^{-1} f_{2}[v, w, \sigma, \mu]^{T} \\
\sigma-\left(v, v_{0}\right)_{L^{2}}+\xi_{R} \\
\mu-\left(w, w_{0}\right)_{L^{2}}+\xi_{I}
\end{array}\right]
$$

is also compact, and the weak problem (13) can be rewritten equivalently in the fixedpoint form

$$
F[v, w, \sigma, \mu]^{T}=[v, w, \sigma, \mu]^{T} .
$$

In the following, for a general map $A$ and a general set $U, A U$ means

$$
A U:=\{A u \mid u \in U\}
$$

Then Schauder's fixed-point theorem asserts that if a nonempty, bounded, convex and closed set $U \subset X$ satisfies

$$
F U \subset U
$$

then there exists a fixed-point of $F$ in $U$.

\section{Finite dimensional subspace and projection error}

In this section, we introduce a finite dimensional approximation subspace $S_{h} \subset H_{0}^{2}(\Omega)$, using basis functions constructed from piecewise cubic Hermite interpolating polynomials, and show a priori error estimates for a projection from $H_{0}^{2}(\Omega)$ onto $S_{h}$.

The interval $\Omega$ is divided into $K$ equal parts:

$$
-1=x_{0}<x_{1}<\cdots<x_{K-1}<x_{K}=1
$$

with nodes $x_{n}=-1+h n(n=0, \ldots, K)$, where $h:=2 / K$. From standard functions $\Phi(x)$ and $\Psi(x)$ defined by

$\Phi(x)=\left\{\begin{array}{cr}(x+1)^{2}(1-2 x) & -1 \leq x \leq 0 \\ (x-1)^{2}(1+2 x) & 0 \leq x \leq 1 \\ 0 & \text { otherwise, }\end{array} \quad \Psi(x)=\left\{\begin{array}{cc}x(x+1)^{2} & -1 \leq x \leq 0 \\ x(1-x)^{2} & 0 \leq x \leq 1 \\ 0 & \text { otherwise }\end{array}\right.\right.$ 
take

$$
\phi_{n}(x):=\Phi\left(h^{-1}(x+1)-n\right), \quad \psi_{n}(x):=h \Psi\left(h^{-1}(x+1)-n\right) \quad n=1, \ldots, K-1 .
$$

Then these functions satisfy

$\phi_{n}\left(x_{m}\right)=\delta_{n m}, \quad \phi_{n}^{\prime}\left(x_{m}\right)=0, \quad \psi_{n}\left(x_{m}\right)=0, \quad \psi_{n}^{\prime}\left(x_{m}\right)=\delta_{n m}, \quad 1 \leq n \leq K-1, \quad 0 \leq m \leq K$.

We define an approximation subspace $S_{h} \subset H_{0}^{2}(\Omega)$ as

$$
S_{h}:=\operatorname{span}\left\{\phi_{n}, \psi_{n} \mid n=1, \ldots, K-1\right\} .
$$

By the well-definedness of the piecewise cubic Hermite interpolation, an interpolation operator

$$
\mathcal{I}_{H}: H_{0}^{2}(\Omega) \longrightarrow S_{h}
$$

can be defined by

$$
\mathcal{I}_{H} f\left(x_{j}\right)=f\left(x_{j}\right), \quad\left(\mathcal{I}_{H} f\right)^{\prime}\left(x_{j}\right)=f^{\prime}\left(x_{j}\right), \quad 1 \leq j \leq K-1,
$$

and the following error estimates of interpolation:

$$
\begin{aligned}
\left\|\left(f-\mathcal{I}_{H} f\right)^{\prime \prime}\right\| & \leq \pi^{-2} h^{2}\left\|f^{(i v)}\right\|, \\
\left\|\left(f-\mathcal{I}_{H} f\right)^{\prime}\right\| & \leq \pi^{-3} h^{3}\left\|f^{(i v)}\right\|, \\
\left\|f-\mathcal{I}_{H} f\right\| & \leq \pi^{-4} h^{4}\left\|f^{(i v)}\right\|
\end{aligned}
$$

hold for all $f \in H^{4}(\Omega) \cap H_{0}^{2}(\Omega)[8]$.

Next, let $P_{h}: H_{0}^{2}(\Omega) \longrightarrow S_{h}$ be the orthogonal projection defined by

$$
\left(\tilde{\Delta}\left(v-P_{h} v\right), \tilde{\Delta} v_{h}\right)_{L^{2}}=0, \quad \forall v_{h} \in S_{h},
$$

then $P_{h}$ has the following property.

Lemma 1 For all $g \in L^{2}(\Omega)$, the difference between the solution $\omega$ of eq.(16) and its projection $P_{h} \omega$ satisfies constructive a priori estimates

$$
\begin{gathered}
\left\|\omega-P_{h} \omega\right\|_{\tilde{\Delta}} \leq C\|g\|, \\
\left\|\omega-P_{h} \omega\right\| \leq C^{2}\|g\|,
\end{gathered}
$$

where

$$
C:=\frac{\sqrt{3}}{\pi^{2}} h^{2}\left(1+\frac{a^{2}}{\pi^{2}} h^{2}\right) .
$$

Proof. From estimates (18)-(20), we have

$$
\begin{aligned}
\left\|\omega-P_{h} \omega\right\|_{\tilde{\Delta}} & \leq\left\|\omega-\mathcal{I}_{H} \omega\right\|_{\tilde{\Delta}} \\
& =\left(\left\|\omega^{\prime \prime}-\mathcal{I}_{H} \omega^{\prime \prime}\right\|^{2}+2 a^{2}\left\|\omega^{\prime}-\mathcal{I}_{H} \omega^{\prime}\right\|^{2}+a^{4}\left\|\omega-\mathcal{I}_{H} \omega\right\|^{2}\right)^{\frac{1}{2}} \\
& \leq h^{2} \pi^{-2}\left(1+a^{2} h^{2} \pi^{-2}\right)\left\|\omega^{(i v)}\right\|,
\end{aligned}
$$


then (22) follows from $\left\|\omega^{(i v)}\right\| \leq \sqrt{3}\left\|\tilde{\Delta}^{2} \omega\right\|$. This inequality can be obtained by partial integration for $f \in H^{4}(\Omega) \cap H_{0}^{2}(\Omega)$,

$$
\begin{aligned}
\left\|\tilde{\Delta}^{2} f\right\|^{2}= & \left(f^{(i v)}, f^{(i v)}\right)_{L^{2}}-4 a^{2}\left(f^{(i v)}, f^{\prime \prime}\right)_{L^{2}}+2 a^{4}\left(f^{(i v)}, f\right)_{L^{2}}+4 a^{4}\left(f^{\prime \prime}, f^{\prime \prime}\right)_{L^{2}} \\
& -4 a^{6}\left(f^{\prime \prime}, f\right)_{L^{2}}+a^{8}(f, f)_{L^{2}} \\
= & \left(f^{(i v)}, f^{(i v)}\right)_{L^{2}}-4 a^{2}\left(f^{(i v)}, f^{\prime \prime}\right)_{L^{2}}+6 a^{4}\left(f^{\prime \prime}, f^{\prime \prime}\right)_{L^{2}}+4 a^{6}\left(f^{\prime}, f^{\prime}\right)_{L^{2}} \\
& +a^{8}(f, f)_{L^{2}} \\
\geq & \left(f^{(i v)}, f^{(i v)}\right)_{L^{2}}-4 a^{2}\left(f^{(i v)}, f^{\prime \prime}\right)_{L^{2}}+6 a^{4}\left(f^{\prime \prime}, f^{\prime \prime}\right)_{L^{2}},
\end{aligned}
$$

and the inequality

$$
\begin{aligned}
\left(f^{(i v)}, f^{\prime \prime}\right)_{L^{2}} & \leq\left\|f^{(i v)}\right\|\left\|f^{\prime \prime}\right\| \\
& \leq \frac{1}{2}\left(\frac{1}{3 a^{2}}\left\|f^{(i v)}\right\|^{2}+3 a^{2}\left\|f^{\prime \prime}\right\|^{2}\right) .
\end{aligned}
$$

The $L^{2}$-estimate (23) is derived by the usual Aubin-Nitsche technique.

\section{Verification condition}

\subsection{Computable algorithm}

In this section, we propose a computable algorithm constructing a candidate set which is expected to satisfy a sufficient condition for Schauder's fixed-point theorem. Basically, this verification method is an extension of the one for solutions of second-order elliptic boundary value problems introduced by a part of the authors [5].

From now on, the identity maps on $X, S_{h}$ and $H_{0}^{2}(\Omega)$ are denoted by the same symbol I. Define the finite dimensional subspace $X_{h}$ of $X$ by

$$
X_{h}=S_{h} \times S_{h} \times \mathbb{R} \times \mathbb{R}
$$

and the projection $\hat{P}_{h}$ from $X$ to $X_{h}$ by

$$
\hat{P}_{h}[v, w, \sigma, \mu]^{T}=\left[P_{h} v, P_{h} w, \sigma, \mu\right]^{T}
$$

using $P_{h}$ from (21). Then any element $u=[v, w, \mu, \sigma]^{T} \in X$ can be uniquely decomposed into

$$
[v, w, \mu, \sigma]^{T}=[\hat{v}, \hat{w}, \mu, \sigma]^{T}+\left[v_{*}, w_{*}, 0,0\right]^{T}, \quad[\hat{v}, \hat{w}, \mu, \sigma]^{T} \in X_{h}, \quad\left[v_{*}, w_{*}, 0,0\right]^{T} \in X_{*},
$$

where

$X_{*}:=\left\{\left[v_{*}, w_{*}, 0,0\right] \in X \mid v_{*}=\left(I-P_{h}\right) v, w_{*}=\left(I-P_{h}\right) w, v \in H_{0}^{2}(\Omega), w \in H_{0}^{2}(\Omega)\right\} \subset X$.

Therefore, the fixed-point equation $u=F u$ on $X$ is equivalently rewritten as

$$
\left\{\begin{aligned}
\hat{P}_{h} u & =\hat{P}_{h} F u \\
\left(I-\hat{P}_{h}\right) u & =\left(I-\hat{P}_{h}\right) F u .
\end{aligned}\right.
$$


Now, we take an approximate solution $u_{h}=\left[v_{h}, w_{h}, \sigma_{h}, \mu_{h}\right]^{T} \in X_{h}$ obtained by some appropriate numerical method and, in order to accelerate contraction, apply a Newtonlike method to the finite dimensional part in eq.(25). Let us define the Newton-like operator $\mathcal{N}_{h}: X \longrightarrow X_{h}$ by

$$
\mathcal{N}_{h} u:=\hat{P}_{h} u-\left[I-\hat{P}_{h} F^{\prime}\left(u_{h}\right)\right]_{h}^{-1} \hat{P}_{h}(I-F) u .
$$

Here $\left[I-\hat{P}_{h} F^{\prime}\left(u_{h}\right)\right]_{h}^{-1}: X_{h} \longrightarrow X_{h}$ means the inverse of the restriction of the operator $\hat{P}_{h}\left(I-F^{\prime}\left(u_{h}\right)\right): X \longrightarrow X_{h}$ to $X_{h}$, where $F^{\prime}$ denotes the Fréchet derivative of $F$. Note that the existence of $\left[I-\hat{P}_{h} F^{\prime}\left(u_{h}\right)\right]_{h}^{-1}$ is equivalent to the invertibility of a matrix, which is numerically checked in the actual verified computations. Since $\hat{P}_{h} u=\hat{P}_{h} \mathcal{N}_{h} u \Leftrightarrow \hat{P}_{h} u=$ $\hat{P}_{h} F u$, using a compact map $T$ on $X$ defined by

$$
T u=\mathcal{N}_{h} u+\left(I-\hat{P}_{h}\right) F u,
$$

we find that the two fixed-point problems: $u=F u$ and $u=T u$ are equivalent.

Next, for positive constants $\gamma, \delta, c_{1}, c_{2}, \alpha$ and $\beta$, set

$$
\begin{aligned}
U_{h} & :=\left\{\left[\hat{v}_{h}, \hat{w}_{h}, \hat{\sigma}, \hat{\mu}\right]^{T} \in X_{h}\left|\left\|\hat{v}_{h}\right\|_{\tilde{\Delta}} \leq \gamma,\left\|\hat{w}_{h}\right\|_{\tilde{\Delta}} \leq \delta,\right| \hat{\sigma}\left|\leq c_{1},\right| \hat{\mu} \mid \leq c_{2}\right\} \subset X_{h}, \\
U_{*} & :=\left\{\left[v_{*}, w_{*}, 0,0\right]^{T} \in X_{*} \mid\left\|v_{*}\right\|_{\tilde{\Delta}} \leq \alpha,\left\|w_{*}\right\|_{\tilde{\Delta}} \leq \beta,\right\} \subset X_{*},
\end{aligned}
$$

and define a candidate set $U \subset X$ by

$$
U:=u_{h}+U_{h}+U_{*} .
$$

Then a sufficient condition for the fixed-point theorem is as follows.

Theorem 1 When the two inclusions:

$$
\left\{\begin{aligned}
\mathcal{N}_{h} U-u_{h} & \subset U_{h} \\
\left(I-\hat{P}_{h}\right) F U & \subset U_{*}
\end{aligned}\right.
$$

hold, there exists a fixed-point of $T$ in $U$.

Proof. By definition, $U$ is a non-empty, closed, convex and bounded set in $X$. For any $u \in U, \mathcal{N}_{h} u \in X_{h},\left(I-\hat{P}_{h}\right) F u \in X_{*}$, and the decomposition $T u=\mathcal{N}_{h} u+\left(I-\hat{P}_{h}\right) F u$ is unique. Hence by (26), we get $\mathcal{N}_{h} U+\left(I-\hat{P}_{h}\right) F U \subset u_{h}+U_{h}+U_{*}$ in $X$, namely, $T U \subset U$. Therefore, by the compactness of the operator $T$ and Schauder's fixed-point theorem, the desired result is obtained.

We now desribe a procedure to construct the candidate set $U$ of $X$ which is expected to satisfy the inclusion (26). Setting

$$
\mathcal{N}_{h} U-u_{h}=:\left[V_{h}, W_{h}, \Sigma, M\right]^{T} \subset X_{h},
$$

the finite dimensional part of the inclusion, $\mathcal{N}_{h} U-u_{h} \subset U_{h}$, can be written as

$$
\sup _{\bar{v}_{h} \in V_{h}}\left\|\bar{v}_{h}\right\|_{\tilde{\Delta}} \leq \gamma, \quad \sup _{\bar{w}_{h} \in W_{h}}\left\|\bar{w}_{h}\right\|_{\tilde{\Delta}} \leq \delta, \quad \sup _{\bar{\sigma} \in \Sigma}|\bar{\sigma}| \leq c_{1}, \quad \sup _{\bar{\mu} \in M}|\bar{\mu}| \leq c_{2} .
$$


Details of the underlying computations will be explained in Subsection 4.1.

On the other hand, the infinite dimensional part of the inclusion, $\left(I-\hat{P}_{h}\right) F U \subset U_{*}$, means

$$
\left[\begin{array}{c}
\left(I-P_{h}\right)\left(\tilde{\Delta}^{2}\right)^{-1} f_{1}[v, w, \sigma, \mu]^{T} \\
\left(I-P_{h}\right)\left(\tilde{\Delta}^{2}\right)^{-1} f_{2}[v, w, \sigma, \mu]^{T} \\
0 \\
0
\end{array}\right] \subset U_{*}
$$

for any $u \in U$ such that $u=[v, w, \sigma, \mu]^{T}$. Setting

$$
\hat{v}_{*}:=\left(I-P_{h}\right)\left(\tilde{\Delta}^{2}\right)^{-1} f_{1}[v, w, \sigma, \mu]^{T}, \quad \hat{w}_{*}:=\left(I-P_{h}\right)\left(\tilde{\Delta}^{2}\right)^{-1} f_{2}[v, w, \sigma, \mu]^{T},
$$

Lemma 1 assures

$$
\left\|\hat{v}_{*}\right\|_{\tilde{\Delta}} \leq C\left\|f_{1}(u)\right\|, \quad\left\|\hat{w}_{*}\right\|_{\tilde{\Delta}} \leq C\left\|f_{2}(u)\right\|, \quad\left\|\hat{v}_{*}\right\| \leq C^{2}\left\|f_{1}(u)\right\|, \quad\left\|\hat{w}_{*}\right\| \leq C^{2}\left\|f_{2}(u)\right\| .
$$

Therefore, in order to satisfy $\left(I-\hat{P}_{h}\right) F U \subset U_{*}$, the conditions

$$
C \sup _{\bar{u} \in U}\left\|f_{1}(\bar{u})\right\| \leq \alpha, \quad C \sup _{\bar{u} \in U}\left\|f_{2}(\bar{u})\right\| \leq \beta
$$

are sufficient. Note that $C$ defined in (24) is small when $h$ is chosen small.

From this we can derive the following theorem.

Theorem 2 With the notations defined before, if one can check the conditions:

$$
\begin{aligned}
& \sup _{\bar{v}_{h} \in V_{h}}\left\|\bar{v}_{h}\right\|_{\tilde{\Delta}} \leq \gamma, \leq \bar{w}_{h} \|_{\tilde{\Delta}} \leq \delta, \\
& \sup _{\bar{w}_{h} \in W_{h}} \| \bar{w}_{1}, \sup _{\bar{\sigma} \in \Sigma}|\bar{\sigma}| \leq c_{2}, \\
& \sup _{\bar{\mu} \in M}|\bar{\mu}| \leq c_{2} \\
& C \sup _{\bar{u} \in U}\left\|f_{1}(\bar{u})\right\| \leq \alpha, \\
& C \sup _{\bar{u} \in U}\left\|f_{2}(\bar{u})\right\| \leq \beta,
\end{aligned}
$$

then there exists fixed-point of $T$ in $U$.

Based on Theorem 2, we propose a verification algorithm in Figure 2.

The extension procedure involving $\varepsilon$ occurring in this algorithm is called " $\varepsilon$-inflation" which is a kind of acceleration technique. The concrete value of $\varepsilon>0$ should be adapted to the actual problem. Experimentally, the initial values of $\gamma^{(0)}, \delta^{(0)}, c_{1}^{(0)}, c_{2}^{(0)}, \alpha^{(0)}$ and $\beta^{(0)}$ are taken as machine epsilon. 


\section{Verification algorithm}

- $k=0$

Set initial values $\gamma^{(0)}, \delta^{(0)}, c_{1}^{(0)}, c_{2}^{(0)}, \alpha^{(0)}, \beta^{(0)}>0$.

- $k \geq 1$

1. For a fixed small constant $\varepsilon>0$ set

$$
\begin{aligned}
& \hat{\gamma}^{(k)}:=(1+\varepsilon) \gamma^{(k-1)}, \quad \quad \hat{\delta}^{(k)}:=(1+\varepsilon) \delta^{(k-1)}, \quad \hat{c}_{1}^{(k)}:=(1+\varepsilon) c_{1}^{(k-1)}, \\
& {\hat{c_{2}}}^{(k)}:=(1+\varepsilon) c_{2}^{(k-1)}, \quad \hat{\alpha}^{(k)}:=(1+\varepsilon) \alpha^{(k-1)}, \quad \hat{\beta}^{(k)}:=(1+\varepsilon) \beta^{(k-1)} \text {. }
\end{aligned}
$$

2. The $k$-th candidate set $U^{(k)}$ is defined by

$$
\begin{aligned}
U_{h}^{(k)} & :=\left\{\left[\hat{v}_{h}, \hat{w}_{h}, \hat{\sigma}, \hat{\mu}\right]^{T} \in X_{h}\left|\left\|\hat{v}_{h}\right\|_{\tilde{\Delta}} \leq \hat{\gamma}^{(k)},\left\|\hat{w}_{h}\right\|_{\tilde{\Delta}} \leq \hat{\delta}^{(k)},\right| \hat{\sigma}\left|\leq{\hat{c_{1}}}^{(k)},\right| \hat{\mu} \mid \leq{\hat{c_{2}}}^{(k)}\right\}, \\
U_{*}^{(k)} & :=\left\{\left[v_{*}, w_{*}, 0,0\right]^{T} \in X_{*} \mid\left\|v_{*}\right\|_{\tilde{\Delta}} \leq \hat{\alpha}^{(k)},\left\|w_{*}\right\|_{\tilde{\Delta}} \leq \hat{\beta}^{(k)},\right\}, \\
U^{(k)} & :=u_{h}+U_{h}^{(k)}+U_{*}^{(k)} .
\end{aligned}
$$

3. Evaluate $N_{h} U^{(k)}-u_{h} \subset X_{h}$ as

$$
\left[V_{h}^{(k)}, W_{h}^{(k)}, \Sigma^{(k)}, M^{(k)}\right]^{T}:=\mathcal{N}_{h} U^{(k)}-u_{h} .
$$

4. Compute values of the $k$-th iteration by

$$
\begin{aligned}
\gamma^{(k)} & :=\sup _{\bar{v}_{h} \in V_{h}^{(k)}}\left\|\bar{v}_{h}\right\|_{\tilde{\Delta}}, \\
\delta^{(k)} & :=\sup _{\bar{w}_{h} \in W_{h}^{(k)}}\left\|\bar{w}_{h}\right\|_{\tilde{\Delta}}, \\
c_{1}^{(k)} & :=\sup _{\bar{\sigma} \in \Sigma^{(k)}}|\bar{\sigma}|, \\
c_{2}^{(k)} & :=\sup _{\bar{\mu} \in M^{(k)}}|\bar{\mu}|, \\
\alpha^{(k)} & :=C \sup _{\bar{u} \in U^{(k)}}\left\|f_{1}(\bar{u})\right\|, \\
\beta^{(k)} & :=C \sup _{\bar{u} \in U^{(k)}}\left\|f_{2}(\bar{u})\right\| .
\end{aligned}
$$

5. If $\gamma^{(k)} \leq \hat{\gamma}^{(k)}, \delta^{(k)} \leq \hat{\delta}^{(k)}, c_{1}^{(k)} \leq{\hat{c_{1}}}^{(k)}, c_{2}^{(k)} \leq{\hat{c_{2}}}^{(k)}, \alpha^{(k)} \leq \hat{\alpha}^{(k)}, \beta^{(k)} \leq \hat{\beta}^{(k)}$ hold then stop, and there exists a desired solution in $U^{(k)} \subset X$.

6 . Set $k:=k+1$ and return to the step 1 . If $k$ reaches a maximum iteration number or some values exceed a criterion then stop, and the verification fails.

Figure 2: 


\subsection{Detailed computation}

Omitting iteration numbers' notation, in the verification step, given 6 parameters $\alpha, \beta, \gamma, \delta, c_{1}$ and $c_{2}>0$, we have to compute

$$
\begin{array}{ll}
\hat{\gamma}=\sup _{\bar{v}_{h} \in V_{h}}\left\|\bar{v}_{h}\right\|_{\tilde{\Delta}}, & \hat{\delta}=\sup _{\bar{w}_{h} \in W_{h}}\left\|\bar{w}_{h}\right\|_{\tilde{\Delta}}, \\
\hat{c_{1}}=\sup _{\bar{\sigma} \in \Sigma}|\bar{\sigma}|, & \hat{c_{2}}=\sup _{\bar{\mu} \in M}|\bar{\mu}|, \\
\hat{\alpha}=C \sup _{\bar{u} \in U}\left\|f_{1}(\bar{u})\right\|, & \hat{\beta}=C \sup _{\bar{u} \in U}\left\|f_{2}(\bar{u})\right\|,
\end{array}
$$

and confirm

$$
\hat{\alpha} \leq \alpha, \quad \hat{\beta} \leq \beta, \quad \hat{\gamma} \leq \gamma, \quad \hat{\delta} \leq \delta, \quad \hat{c_{1}} \leq c_{1}, \quad \hat{c_{2}} \leq c_{2}
$$

In the actual computation, the candidate set $U$ contains the infinite dimensional term $U_{*}$. Moreover, it is impossible to avoid the effect of rounding error of floating point arithmetic. However, by norm estimates, and interval arithmetic software taking into account effects of rounding error, we can obtain mathematically rigorous upper bounds for $\hat{\gamma}, \hat{\delta}, \hat{c_{1}}, \hat{c_{2}}, \hat{\alpha}$ and $\hat{\beta}$ with possible over-estimates. Let us describe these computations in more detail.

For any $u \in U$ such that

$$
\begin{aligned}
u & =u_{h}+\hat{u}_{h}+u_{*}, \quad \hat{u}_{h} \in U_{h}, \quad u_{*} \in U_{*} \\
& =\left[\begin{array}{c}
v_{h} \\
w_{h} \\
\sigma_{h} \\
\mu_{h}
\end{array}\right]+\left[\begin{array}{c}
\hat{v}_{h} \\
\hat{w}_{h} \\
\hat{\sigma} \\
\hat{\mu}
\end{array}\right]+\left[\begin{array}{c}
v_{*} \\
w_{*} \\
0 \\
0
\end{array}\right]
\end{aligned}
$$

after some calculations we obtain

$$
\begin{aligned}
\mathcal{N}_{h} u-u_{h}=\left[I-\hat{P}_{h} F^{\prime}\left(u_{h}\right)\right]_{h}^{-1}\left[\begin{array}{c}
-v_{h}+P_{h}\left(\tilde{\Delta}^{2}\right)^{-1} f_{1}\left(u_{h}\right) \\
-w_{h}+P_{h}\left(\tilde{\Delta}^{2}\right)^{-1} f_{2}\left(u_{h}\right) \\
\xi_{R}-\left(v_{h}, v_{0}\right)_{L^{2}} \\
\xi_{I}-\left(w_{h}, w_{0}\right)_{L^{2}}
\end{array}\right] \\
+\left[I-\hat{P}_{h} F^{\prime}\left(u_{h}\right)\right]_{h}^{-1}\left[\begin{array}{c}
P_{h}\left(\tilde{\Delta}^{2}\right)^{-1}\left\{\hat{\sigma} \tilde{\Delta} \hat{v}_{h}-\hat{\mu} \tilde{\Delta} \hat{w}_{h}+f_{1}\left[v_{*}, w_{*}, \sigma_{h}+\hat{\sigma}, \mu_{h}+\hat{\mu}\right]^{T}\right\} \\
P_{h}\left(\tilde{\Delta}^{2}\right)^{-1}\left\{\hat{\sigma} \tilde{\Delta} \hat{w}_{h}+\hat{\mu} \tilde{\Delta} \hat{v}_{h}+f_{2}\left[v_{*}, w_{*}, \sigma_{h}+\hat{\sigma}, \mu_{h}+\hat{\mu}\right]^{T}\right\} \\
-\left(v_{*}, v_{0}\right)_{L^{2}} \\
-\left(w_{*}, w_{0}\right)_{L^{2}}
\end{array}\right] .
\end{aligned}
$$

The first term

$$
\left[\begin{array}{c}
r_{1} \\
r_{2} \\
r_{3} \\
r_{4}
\end{array}\right]:=\left[I-\hat{P}_{h} F^{\prime}\left(u_{h}\right)\right]_{h}^{-1}\left[\begin{array}{c}
-v_{h}+P_{h}\left(\tilde{\Delta}^{2}\right)^{-1} f_{1}\left(u_{h}\right) \\
-w_{h}+P_{h}\left(\tilde{\Delta}^{2}\right)^{-1} f_{2}\left(u_{h}\right) \\
\xi_{R}-\left(v_{h}, v_{0}\right)_{L^{2}} \\
\xi_{I}-\left(w_{h}, w_{0}\right)_{L^{2}}
\end{array}\right] \in X_{h}
$$


on the right-hand side of eq.(27) is only constructed by the approximate solution and known functions; note that $P_{h}\left(\tilde{\Delta}^{2}\right)^{-1} f_{i}\left(u_{h}\right)$ is the solution of a finite dimensional linear problem. Therefore, each norm

$$
\left\|r_{1}\right\|_{\tilde{\Delta}}, \quad\left\|r_{2}\right\|_{\tilde{\Delta}}, \quad\left|r_{3}\right|, \quad \text { and } \quad\left|r_{4}\right|
$$

can be bounded by solving some linear algebraic systems with interval arithmetic.

On the other hand, when we set

$$
\left[t_{1}, t_{2}, t_{3}, t_{4}\right]^{T}=\left[I-\hat{P}_{h} F^{\prime}\left(u_{h}\right)\right]_{h}^{-1}\left[s_{1}, s_{2}, s_{3}, s_{4}\right]^{T} \in X_{h},
$$

for $\left[s_{1}, s_{2}, s_{3}, s_{4}\right]^{T} \in X_{h}$, it can be shown that

$$
\left\{\begin{aligned}
\left\|t_{1}\right\|_{\tilde{\Delta}} & \leq \rho_{1}\left\|s_{1}\right\|_{\tilde{\Delta}}+\rho_{2}\left\|s_{2}\right\|_{\tilde{\Delta}}+\left\|L^{T}\left(G_{13}^{-1} s_{3}+G_{14}^{-1} s_{4}\right)\right\|_{E}, \\
\left\|t_{2}\right\|_{\tilde{\Delta}} & \leq \rho_{3}\left\|s_{1}\right\|_{\tilde{\Delta}}+\rho_{4}\left\|s_{2}\right\|_{\tilde{\Delta}}+\left\|L^{T}\left(G_{23}^{-1} s_{3}+G_{24}^{-1} s_{4}\right)\right\|_{E}, \\
\left|t_{3}\right| & \leq \rho_{5}\left\|s_{1}\right\|_{\tilde{\Delta}}+\rho_{6}\left\|s_{2}\right\|_{\tilde{\Delta}}+\left|G_{33}^{-1} s_{3}+G_{34}^{-1} s_{4}\right|, \\
\left|t_{4}\right| & \leq \rho_{7}\left\|s_{1}\right\|_{\tilde{\Delta}}+\rho_{8}\left\|s_{2}\right\|_{\tilde{\Delta}}+\left|G_{43}^{-1} s_{3}+G_{44}^{-1} s_{4}\right|,
\end{aligned}\right.
$$

where

$$
\begin{gathered}
G:=\left[\begin{array}{cccc}
A_{1}-\sigma_{h} A_{3} & -A_{2}+\mu_{h} A_{3} & -A_{3} \boldsymbol{v}_{h} & A_{3} \boldsymbol{w}_{h} \\
A_{2}-\mu_{h} A_{3} & A_{1}-\sigma_{h} A_{3} & -A_{3} \boldsymbol{w}_{h} & -A_{3} \boldsymbol{v}_{h} \\
\boldsymbol{v}_{0}^{T} A_{4} & 0 & 0 & 0 \\
0 & \boldsymbol{w}_{0}^{T} A_{4} & 0 & 0
\end{array}\right] \in \mathbb{R}^{2 K \times 2 K}, \\
{\left[A_{1}\right]_{n m}:=\left(\tilde{\Delta} \hat{\phi}_{m}, \tilde{\Delta} \hat{\phi}_{n}\right)_{L^{2}}} \\
{\left[A_{2}\right]_{n m}:=a R\left(\left(V \tilde{\Delta}+V^{\prime \prime}\right) \hat{\phi}_{m}, \hat{\phi}_{n}\right)_{L^{2}},} \\
{\left[A_{3}\right]_{n m}:=\left(\tilde{\Delta} \hat{\phi}_{m}, \hat{\phi}_{n}\right)_{L^{2}}} \\
{\left[A_{4}\right]_{n m}:=\left(\hat{\phi}_{m}, \hat{\phi}_{n}\right)_{L^{2}},}
\end{gathered}
$$

$L$ is the Cholesky factor of $A_{1}: A_{1}=L L^{T}$,

$$
\begin{gathered}
C_{1}:=\sqrt{\frac{\lambda_{\max }\left(A_{4}\right)}{\lambda_{\min }\left(A_{1}\right)}}, \quad G^{-1}=:\left[\begin{array}{llll}
G_{11}^{-1} & G_{12}^{-1} & G_{13}^{-1} & G_{14}^{-1} \\
G_{21}^{-1} & G_{22}^{-1} & G_{23}^{-1} & G_{24}^{-1} \\
G_{31}^{-1} & G_{32}^{-1} & G_{33}^{-1} & G_{34}^{-1} \\
G_{41}^{-1} & G_{42}^{-1} & G_{43}^{-1} & G_{44}^{-1}
\end{array}\right], \\
\rho_{1}:=\left\|L^{T} G_{11}^{-1} L\right\|_{M}, \quad \rho_{2}:=\left\|L^{T} G_{12}^{-1} L\right\|_{M}, \quad \rho_{3}:=\left\|L^{T} G_{21}^{-1} L\right\|_{M}, \quad \rho_{4}:=\left\|L^{T} G_{22}^{-1} L\right\|_{M}, \\
\rho_{5}:=\left\|\left(G_{31}^{-1} L\right)^{T}\right\|_{E}, \quad \rho_{6}:=\left\|\left(G_{32}^{-1} L\right)^{T}\right\|_{E}, \quad \rho_{7}:=\left\|\left(G_{41}^{-1} L\right)^{T}\right\|_{E}, \quad \rho_{8}:=\left\|\left(G_{42}^{-1} L\right)^{T}\right\|_{E},
\end{gathered}
$$

and $\|\cdot\|_{M}$ and $\|\cdot\|_{E}$ mean the usual matrix and vector 2-norms. Evaluations of $\rho_{1}, \rho_{2}, \rho_{3}$ and $\rho_{4}$ can be reduced to the computation of the maximum singular value of a matrix. 
Therefore, norm bounds for the second term on the right-hand side of eq.(27) are obtained from norm bounds for

$$
\left[\begin{array}{c}
s_{1} \\
s_{2} \\
s_{3} \\
s_{4}
\end{array}\right]:=\left[\begin{array}{c}
P_{h}\left(\tilde{\Delta}^{2}\right)^{-1}\left\{\sigma \tilde{\Delta} \hat{v}_{h}-\mu \tilde{\Delta} \hat{w}_{h}+f_{1}\left[v_{*}, w_{*}, \sigma_{h}+\hat{\sigma}, \mu_{h}+\hat{\mu}\right]^{T}\right\} \\
P_{h}\left(\tilde{\Delta}^{2}\right)^{-1}\left\{\sigma \tilde{\Delta} \hat{w}_{h}+\mu \tilde{\Delta} \hat{v}_{h}+f_{2}\left[v_{*}, w_{*}, \sigma_{h}+\hat{\sigma}, \mu_{h}+\hat{\mu}\right]^{T}\right\} \\
-\left(v_{*}, v_{0}\right)_{L^{2}} \\
-\left(w_{*}, w_{0}\right)_{L^{2}}
\end{array}\right]
$$

which in turn can be computed as

$$
\begin{aligned}
\left\|s_{1}\right\|_{\tilde{\Delta}} & \leq C_{1}\left(\hat{c_{1}} \hat{\gamma}+\hat{c_{2}} \hat{\delta}\right)+C\left(\left(\rho_{9}+\tau_{2}\right) \hat{\beta}+\tau_{1} \hat{\alpha}\right), \\
\left\|s_{2}\right\|_{\tilde{\Delta}} & \leq C_{1}\left(\hat{c_{1}} \hat{\delta}+\hat{c_{2}} \hat{\gamma}\right)+C\left(\left(\rho_{9}+\tau_{2}\right) \hat{\alpha}+\tau_{1} \hat{\beta}\right), \\
\left|s_{3}\right| & \leq C \hat{\alpha} \rho_{10}, \\
\left|s_{4}\right| & \leq C \hat{\beta} \rho_{11},
\end{aligned}
$$

where

$$
\begin{gathered}
\tau_{1}:=\sup _{|\hat{\sigma}| \leq \hat{c_{1}}}\left|\sigma_{h}+\hat{\sigma}\right|, \quad \tau_{2}:=\sup _{|\hat{\mu}| \leq \hat{c_{2}}}\left|\mu_{h}+\hat{\mu}\right|, \\
\rho_{9}:=a R\|V\|_{\infty}+\sqrt{2} R\left\|V^{\prime}\right\|_{\infty}+\frac{2 R}{a}\left\|V^{\prime \prime}\right\|_{\infty}, \quad \rho_{10}:=\left\|v_{0}\right\|, \quad \rho_{11}:=\left\|w_{0}\right\| .
\end{gathered}
$$

Moreover, estimates for $\left\|f_{1}(u)\right\|$ and $\left\|f_{2}(u)\right\|$ are obtained by

$$
\begin{aligned}
& \left\|f_{1}(u)\right\| \leq \rho_{12}+\tau_{3} \hat{\delta}+\rho_{13} C_{1} \hat{\delta}+\tau_{1} \hat{\gamma}+\tau_{4}+\tau_{3} \hat{\beta}+\rho_{13} C \hat{\beta}+\tau_{1} \hat{\alpha} \\
& \left\|f_{2}(u)\right\| \leq \rho_{14}+\tau_{3} \hat{\gamma}+\rho_{13} C_{1} \hat{\gamma}+\tau_{1} \hat{\delta}+\tau_{5}+\tau_{3} \hat{\alpha}+\rho_{13} C \hat{\alpha}+\tau_{1} \hat{\beta}
\end{aligned}
$$

where

$$
\begin{aligned}
& \tau_{3}:=\sup _{|\hat{\mu}| \leq \hat{c_{2}}}\left\|a R V-\hat{\mu}_{h}-\hat{\mu}\right\|_{\infty} \\
& \tau_{4}:=\sup _{|\hat{\sigma}| \leq \hat{c_{1}},|\hat{\mu}| \leq \hat{c_{2}}}\left\|\hat{\sigma} v_{h}-\hat{\mu} w_{h}\right\|_{\tilde{\Delta}}, \\
& \tau_{5}:=\sup _{|\hat{\sigma}| \leq \hat{c_{1}},|\hat{\mu}| \leq \hat{c_{2}}}\left\|\hat{\sigma} w_{h}+\hat{\mu} v_{h}\right\|_{\tilde{\Delta}} \\
& \rho_{12}:=\left\|f_{1}\left(u_{h}\right)\right\|, \quad \rho_{13}:=\left\|a R V^{\prime \prime}\right\|_{\infty}, \quad \rho_{14}:=\left\|f_{2}\left(u_{h}\right)\right\| .
\end{aligned}
$$

\section{$5 \quad$ Verification results}

We now show some verification results. It is well known that the discretization of the Orr-Sommerfeld equation yields a stiff system. The quadruple precision interval arithmetic in each verification step was implemented using Sun ONE Studio 7, Compiler Collection Fortran 95 on FUJITSU PRIMEPOWER850 (CPU: SPARC64-GP 1.3GHz, OS: Solaris8). The approximate solutions were obtained by a Newton-Raphson method using usual floating point arithmetic with quadruple precision. 


\subsection{Result 1}

For $R=5774$ and $a=1.02$, the verification algorithm executed successfully with $K=$ 1000 in the following candidate set:

$$
U=u_{h}+U_{h}+U_{*}, \quad U_{h}=\left[V_{h}, W_{h}, \Sigma, M\right]^{T}, \quad U_{*}=\left[V_{*}, W_{*}, 0,0\right]^{T},
$$

where

$$
\begin{aligned}
& \left\|V_{h}\right\|_{\tilde{\Delta}} \leq 5.518 \times 10^{-4}, \quad\left\|W_{h}\right\|_{\tilde{\Delta}} \leq 5.383 \times 10^{-4}, \\
& \left\|V_{*}\right\|_{\tilde{\Delta}} \leq 3.868 \times 10^{-3}, \quad\left\|W_{*}\right\|_{\tilde{\Delta}} \leq 6.578 \times 10^{-3} .
\end{aligned}
$$

Especially, an eigenvalue can be enclosed within the complex interval

$$
\lambda \in[-0.03745,0.00347]+i[1554.34370,1554.38555] .
$$

\subsection{Result 2}

For $R=5775$ and $a=1.02$, the verification algorithm also executed successfully with $K=1000$ in the following candidate set:

$$
U=u_{h}+U_{h}+U_{*}, \quad U_{h}=\left[V_{h}, W_{h}, \Sigma, M\right]^{T}, \quad U_{*}=\left[V_{*}, W_{*}, 0,0\right]^{T},
$$

where

$$
\begin{aligned}
& \left\|V_{h}\right\|_{\tilde{\Delta}} \leq 5.388 \times 10^{-4}, \quad\left\|W_{h}\right\|_{\tilde{\Delta}} \leq 5.523 \times 10^{-4}, \\
& \left\|V_{*}\right\|_{\tilde{\Delta}} \leq 6.587 \times 10^{-3}, \quad\left\|W_{*}\right\|_{\tilde{\Delta}} \leq 3.867 \times 10^{-3} .
\end{aligned}
$$

Especially, an eigenvalue can be enclosed within the complex interval

$$
\lambda \in[-0.04719,-0.00625]+i[1554.56608,1554.60797]
$$

As mentioned in Section 1, within the frame of linearized stability theory, we can therefore conclude that the flow is unstable because at least one spectral point is located in the left complex half-plane.

Figure 3 shows the minimum Reynolds number $R$ for which the verification algorithm assures that the real part of an eigenvalue is strictly negative for the corresponding wave number $a$. Therefore, it is expected that the critical curve $\operatorname{Re}(\lambda)=0$ should be located below these dots.

\section{Conclusion}

For some fixed Reynolds number and wave number $[a, R]$ we can enclose an eigenpair for the Orr-Sommerfeld equation with Poiseuille flow from hydrodynamic stability. We cannot say for certain whether the enclosed eigenvalue has the smallest real part or not, and we also cannot enclose the critical curve. These questions must be solved in our future work. We wish to remark that in principle, a computer-assisted stability proof could be given with the aid of [3], where a box has been computed which contains all eigenvalues of the Orr-Sommerfeld problem, and which has a compact intersection with the left complex half-plane.

This work was supported by a Grant-in-Aid from the Ministry of Education, Culture, Sports, Science and Technology of Japan (No.18540127, No.15204007, No.16104001). 


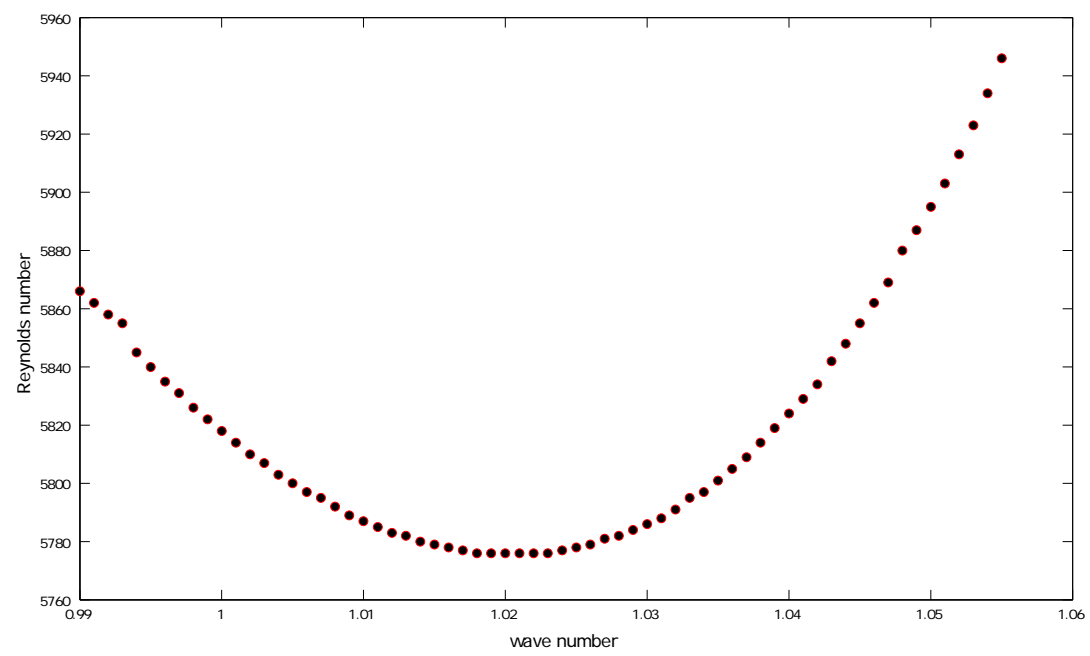

Figure 3: $[a, R]$ with $\operatorname{Re}(\lambda)<0$

\section{References}

[1] Klein, P. P.: Including Eigenvalues of the Plane Orr-Sommerfeld Problem, Applications of Mathematics, Vol.38, No.6, pp.452-458 (1993).

[2] Lahmann, J. and Plum, M., A computer-assisted instability proof for the OrrSommerfeld equation with Blasius profile, ZAMM, Vol.84, pp.188-204 (2004).

[3] Lahmann, J. and Plum, M., On the spectrum of the Orr-Sommerfeld equation on the semiaxis, Mathematische Nachrichten, Vol.216, pp.145-153 (2000).

[4] Lin, C. C.: The Theory of Hydrodynamic Stability, Cambridge University Press, Cambridge, 1955.

[5] Nakao, M. T. and Watanabe, Y., An Efficient Approach to the Numerical Verification for Solutions of Elliptic Differential Equations, Numerical Algorithms, Vol.37, Issue 1-4 (2004), 311-323.

[6] Orr, W. M'F.: The Stability or Instability of the Steady Motions of a Perfect Liquid and of a Viscous Liquid, Part I (A Perfect Liquid), Part II (A Viscous Liquid), Proceedings of the Royal Irish Academy Sect.A, Vol.27, pp.9-138 (1907).

[7] Orszag, S. A.: Accurate Solution of the Orr-Sommerfeld Stability Equation, J. Fluid Mech., Vol.50, Part4, pp.689-703 (1971).

[8] Schultz. M. H.: Spline Analysis, Prentice-Hall, London, 1973.

[9] Sommerfeld, A.: Ein Beitrag zur Hydrodynamischen Erklärung der Turbulenten Flüssigkeitsbewegungen, Atti del IV Congresso Internazionale dei Matematici (Roma, 6-11 Aprile 1908), Vol.3, pp.116-124 (1909). 


\section{List of MHF Preprint Series, Kyushu University \\ 21st Century COE Program \\ Development of Dynamic Mathematics with High Functionality}

MHF2005-1 Hideki KOSAKI

Matrix trace inequalities related to uncertainty principle

MHF2005-2 Masahisa TABATA

Discrepancy between theory and real computation on the stability of some finite element schemes

MHF2005-3 Yuko ARAKI \& Sadanori KONISHI

Functional regression modeling via regularized basis expansions and model selection

MHF2005-4 Yuko ARAKI \& Sadanori KONISHI

Functional discriminant analysis via regularized basis expansions

MHF2005-5 Kenji KAJIWARA, Tetsu MASUDA, Masatoshi NOUMI, Yasuhiro OHTA \& Yasuhiko YAMADA

Point configurations, Cremona transformations and the elliptic difference Painlevé equations

MHF2005-6 Kenji KAJIWARA, Tetsu MASUDA, Masatoshi NOUMI, Yasuhiro OHTA \& Yasuhiko YAMADA

Construction of hypergeometric solutions to the $q$ - Painlevé equations

MHF2005-7 Hiroki MASUDA

Simple estimators for non-linear Markovian trend from sampled data:

I. ergodic cases

MHF2005-8 Hiroki MASUDA \& Nakahiro YOSHIDA

Edgeworth expansion for a class of Ornstein-Uhlenbeck-based models

MHF2005-9 Masayuki UCHIDA

Approximate martingale estimating functions under small perturbations of dynamical systems

MHF2005-10 Ryo MATSUZAKI \& Masayuki UCHIDA

One-step estimators for diffusion processes with small dispersion parameters from discrete observations

MHF2005-11 Junichi MATSUKUBO, Ryo MATSUZAKI \& Masayuki UCHIDA

Estimation for a discretely observed small diffusion process with a linear drift

MHF2005-12 Masayuki UCHIDA \& Nakahiro YOSHIDA

AIC for ergodic diffusion processes from discrete observations 
MHF2005-13 Hiromichi GOTO \& Kenji KAJIWARA

Generating function related to the Okamoto polynomials for the Painlevé IV equation

MHF2005-14 Masato KIMURA \& Shin-ichi NAGATA

Precise asymptotic behaviour of the first eigenvalue of Sturm-Liouville problems with large drift

MHF2005-15 Daisuke TAGAMI \& Masahisa TABATA

Numerical computations of a melting glass convection in the furnace

MHF2005-16 Raimundas VIDŪNAS

Normalized Leonard pairs and Askey-Wilson relations

MHF2005-17 Raimundas VIDŪNAS

Askey-Wilson relations and Leonard pairs

MHF2005-18 Kenji KAJIWARA \& Atsushi MUKAIHIRA

Soliton solutions for the non-autonomous discrete-time Toda lattice equation

MHF2005-19 Yuu HARIYA

Construction of Gibbs measures for 1-dimensional continuum fields

MHF2005-20 Yuu HARIYA

Integration by parts formulae for the Wiener measure restricted to subsets in $\mathbb{R}^{d}$

MHF2005-21 Yuu HARIYA

A time-change approach to Kotani's extension of Yor's formula

MHF2005-22 Tadahisa FUNAKI, Yuu HARIYA \& Mark YOR

Wiener integrals for centered powers of Bessel processes, I

MHF2005-23 Masahisa TABATA \& Satoshi KAIZU

Finite element schemes for two-fluids flow problems

MHF2005-24 Ken-ichi MARUNO \& Yasuhiro OHTA

Determinant form of dark soliton solutions of the discrete nonlinear Schrödinger equation

MHF2005-25 Alexander V. KITAEV \& Raimundas VIDŪNAS

Quadratic transformations of the sixth Painlevé equation

MHF2005-26 Toru FUJII \& Sadanori KONISHI

Nonlinear regression modeling via regularized wavelets and smoothing parameter selection

MHF2005-27 Shuichi INOKUCHI, Kazumasa HONDA, Hyen Yeal LEE, Tatsuro SATO, Yoshihiro MIZOGUCHI \& Yasuo KAWAHARA

On reversible cellular automata with finite cell array 
MHF2005-28 Toru KOMATSU

Cyclic cubic field with explicit Artin symbols

MHF2005-29 Mitsuhiro T. NAKAO, Kouji HASHIMOTO \& Kaori NAGATOU

A computational approach to constructive a priori and a posteriori error estimates for finite element approximations of bi-harmonic problems

MHF2005-30 Kaori NAGATOU, Kouji HASHIMOTO \& Mitsuhiro T. NAKAO

Numerical verification of stationary solutions for Navier-Stokes problems

MHF2005-31 Hidefumi KAWASAKI

A duality theorem for a three-phase partition problem

MHF2005-32 Hidefumi KAWASAKI

A duality theorem based on triangles separating three convex sets

MHF2005-33 Takeaki FUCHIKAMI \& Hidefumi KAWASAKI

An explicit formula of the Shapley value for a cooperative game induced from the conjugate point

MHF2005-34 Hideki MURAKAWA

A regularization of a reaction-diffusion system approximation to the two-phase

Stefan problem

MHF2006-1 Masahisa TABATA

Numerical simulation of Rayleigh-Taylor problems by an energy-stable finite element scheme

MHF2006-2 Ken-ichi MARUNO \& G R W QUISPEL

Construction of integrals of higher-order mappings

MHF2006-3 Setsuo TANIGUCHI

On the Jacobi field approach to stochastic oscillatory integrals with quadratic phase function

MHF2006-4 Kouji HASHIMOTO, Kaori NAGATOU \& Mitsuhiro T. NAKAO

A computational approach to constructive a priori error estimate for finite element approximations of bi-harmonic problems in nonconvex polygonal domains

MHF2006-5 Hidefumi KAWASAKI

A duality theory based on triangular cylinders separating three convex sets in $R^{n}$

MHF2006-6 Raimundas VIDŪNAS

Uniform convergence of hypergeometric series

MHF2006-7 Yuji KODAMA \& Ken-ichi MARUNO

N-Soliton solutions to the DKP equation and Weyl group actions 
MHF2006-8 Toru KOMATSU

Potentially generic polynomial

MHF2006-9 Toru KOMATSU

Generic sextic polynomial related to the subfield problem of a cubic polynomial

MHF2006-10 Shu TEZUKA \& Anargyros PAPAGEORGIOU

Exact cubature for a class of functions of maximum effective dimension

MHF2006-11 Shu TEZUKA

On high-discrepancy sequences

MHF2006-12 Raimundas VIDŪNAS

Detecting persistent regimes in the North Atlantic Oscillation time series

MHF2006-13 Toru KOMATSU

Tamely Eisenstein field with prime power discriminant

MHF2006-14 Nalini JOSHI, Kenji KAJIWARA \& Marta MAZZOCCO

Generating function associated with the Hankel determinant formula for the solutions of the Painlevé IV equation

MHF2006-15 Raimundas VIDŪNAS

Darboux evaluations of algebraic Gauss hypergeometric functions

MHF2006-16 Masato KIMURA \& Isao WAKANO

New mathematical approach to the energy release rate in crack extension

MHF2006-17 Toru KOMATSU

Arithmetic of the splitting field of Alexander polynomial

MHF2006-18 Hiroki MASUDA

Likelihood estimation of stable Lévy processes from discrete data

MHF2006-19 Hiroshi KAWABI \& Michael RÖCKNER

Essential self-adjointness of Dirichlet operators on a path space with Gibbs measures via an SPDE approach

MHF2006-20 Masahisa TABATA

Energy stable finite element schemes and their applications to two-fluid flow problems

MHF2006-21 Yuzuru INAHAMA \& Hiroshi KAWABI

Asymptotic expansions for the Laplace approximations for Itô functionals of Brownian rough paths

MHF2006-22 Yoshiyuki KAGEI

Resolvent estimates for the linearized compressible Navier-Stokes equation in an infinite layer 
MHF2006-23 Yoshiyuki KAGEI

Asymptotic behavior of the semigroup associated with the linearized compressible Navier-Stokes equation in an infinite layer

MHF2006-24 Akihiro MIKODA, Shuichi INOKUCHI, Yoshihiro MIZOGUCHI \& Mitsuhiko FUJIO

The number of orbits of box-ball systems

MHF2006-25 Toru FUJII \& Sadanori KONISHI

Multi-class logistic discrimination via wavelet-based functionalization and model selection criteria

MHF2006-26 Taro HAMAMOTO, Kenji KAJIWARA \& Nicholas S. WITTE

Hypergeometric solutions to the $q$-Painlevé equation of type $(A+A)$

MHF2006-27 Hiroshi KAWABI \& Tomohiro MIYOKAWA

The Littlewood-Paley-Stein inequality for diffusion processes on general metric spaces

MHF2006-28 Hiroki MASUDA

Notes on estimating inverse-Gaussian and gamma subordinators under highfrequency sampling

MHF2006-29 Setsuo TANIGUCHI

The heat semigroup and kernel associated with certain non-commutative harmonic oscillators

MHF2006-30 Setsuo TANIGUCHI

Stochastic analysis and the KdV equation

MHF2006-31 Masato KIMURA, Hideki KOMURA, Masayasu MIMURA, Hidenori MIYOSHI, Takeshi TAKAISHI \& Daishin UEYAMA

Quantitative study of adaptive mesh FEM with localization index of pattern

MHF2007-1 Taro HAMAMOTO \& Kenji KAJIWARA

Hypergeometric solutions to the $q$-Painlevé equation of type $A$

MHF2007-2 Kouji HASHIMOTO, Kenta KOBAYASHI \& Mitsuhiro T. NAKAO

Verified numerical computation of solutions for the stationary Navier-Stokes equation in nonconvex polygonal domains

MHF2007-3 Kenji KAJIWARA, Marta MAZZOCCO \& Yasuhiro OHTA

A remark on the Hankel determinant formula for solutions of the Toda equation

MHF2007-4 Jun-ichi SATO \& Hidefumi KAWASAKI

Discrete fixed point theorems and their application to Nash equilibrium

MHF2007-5 Mitsuhiro T. NAKAO \& Kouji HASHIMOTO

Constructive error estimates of finite element approximations for non-coercive

elliptic problems and its applications 
MHF2007-6 Kouji HASHIMOTO

A preconditioned method for saddle point problems

MHF2007-7 Christopher MALON, Seiichi UCHIDA \& Masakazu SUZUKI

Mathematical symbol recognition with support vector machines

MHF2007-8 Kenta KOBAYASHI

On the global uniqueness of Stokes' wave of extreme form

MHF2007-9 Kenta KOBAYASHI

A constructive a priori error estimation for finite element discretizations in a non-convex domain using singular functions

MHF2007-10 Myoungnyoun KIM, Mitsuhiro T. NAKAO, Yoshitaka WATANABE \& Takaaki NISHIDA

A numerical verification method of bifurcating solutions for 3-dimensional Rayleigh-Bénard problems

MHF2007-11 Yoshiyuki KAGEI

Large time behavior of solutions to the compressible Navier-Stokes equation in an infinite layer

MHF2007-12 Takashi YANAGAWA, Satoshi AOKI and Tetsuji OHYAMA

Human finger vein images are diverse and its patterns are useful for personal identification

MHF2007-13 Masahisa TABATA

Finite element schemes based on energy-stable approximation for two-fluid flow problems with surface tension

MHF2007-14 Mitsuhiro T. NAKAO \& Takehiko KINOSHITA

Some remarks on the behaviour of the finite element solution in nonsmooth domains

MHF2007-15 Yoshiyuki KAGEI \& Takumi NUKUMIZU

Asymptotic behavior of solutions to the compressible Navier-Stokes equation in a cylindrical domain

MHF2007-16 Shuichi INOKUCHI, Yoshihiro MIZOGUCHI, Hyen Yeal LEE \& Yasuo KAWAHARA

Periodic Behaviors of Quantum Cellular Automata

MHF2007-17 Makoto HIROTA\& Yasuhide FUKUMOTO

Energy of hydrodynamic and magnetohydrodynamic waves with point and continuous spectra

MHF2007-18 Mitsunori KAYANO\& Sadanori KONISHI

Functional principal component analysis via regularized Gaussian basis expansions and its application to unbalanced data 
MHF2007-19 Mitsunori KAYANO, Koji DOZONO \& Sadanori KONISHI

Functional Cluster Analysis via Orthonormalized Gaussian Basis Expansions and Its Application

MHF2008-1 Jun-ichi SATO

An application of a discrete fixed point theorem to the Cournot model

MHF2008-2 Kei HIROSE, Shuichi KAWANO, Sadanori KONISHI \& Masanori ICHIKAWA Bayesian factor analysis and model selection

MHF2008-3 Yoshitaka WATANABE, Michael PLUM \& Mitsuhiro T. NAKAO

A computer-assisted instability proof for the Orr-Sommerfeld problem with Poiseuille flow 